\title{
Contactless Planar Actuator with Manipulator: a Motion System without Cables and Physical Contact between the Mover and the Fixed World
}

\author{
Jeroen de Boeij, Elena Lomonova and Jorge Duarte \\ Department of Electrical Engineering \\ Eindhoven University of Technology \\ 5600 MB Eindhoven, The Netherlands \\ Email: j.d.boeij@tue.nl
}

\begin{abstract}
A novel motion system is presented that provides completely contactless operation from the fixed world. It consists of a contactless planar actuator with 6-DOF and long-stroke movement in the $x-y$ plane, with a 2-DOF manipulator on top of the floating platform. The contactless energy transfer (100W continuous) to the moving platform for operating manipulator is integrated into the magnetic suspension. The manipulator is controlled by a controller on the fixed world with a low latency wireless link based on a custom protocol $(<400 \mu$ s additional closed loop delay). The behavior of the system is demonstrated with measurements of the experimental setup.
\end{abstract}

\section{INTRODUCTION}

High-precision machines usually consist of two motion systems i.e. a long stroke system that has micrometer accuracy and a short stroke motion system that realizes the submicron precision. Next to the complex mechanical structure that leads to crosstalk, there are cable slabs between the fixed world and the moving part that induces disturbances such as friction and vibrations. Often times mechanical or air bearings are used, which can also introduce unwanted phenomena in the system.

Ideally, a motion system with long-stroke movement capability without cables to the moving part and without contact with the fixed world should be used. Cables slabs carry both power and information so creating such a system requires 6DOF electromagnetic suspension and propulsion, contactless energy transfer and wireless control.

If parallel processing is required, i.e. movement and positioning in parallel with inspection, calibration, assembling, scanning, measuring, etc., real-time synchronization of all systems is of vital importance, which implies that the communication delay in the wireless control loop needs to be much smaller that the sample time.

To summarize, contactless techniques can greatly improve the flexibility of high-precision machine design. The three contactless techniques that are discussed are:

1) 6-DOF Electromagnetic suspension and propulsion.

2) Contactless energy transfer to the moving parts.

3) Real-time wireless control.

At Eindhoven University of Technology, a prototype has been built that integrates all three techniques into one motion system.

\section{CONTACTLESS Planar ACTUATOR With MANIPULATOR}

Combining these three techniques results in a contactless planar actuator [1], [2], [3], [6] with manipulator, of which a schematic representation is shown in Fig. 1. The platform is suspended and propelled by magnetic bearings, with moving magnets and stationary coils (contactless planar actuator). The energy to operate the manipulator is provided by contactless energy transfer (CET), which can continuously supply power while the platform is moving by means of inductive coupling between stationary primary coils and a moving secondary coil [8].

The communication between the ground controller and the manipulator is done by a low-latency wireless link (RX/TX), with a custom protocol. Using this wireless link, the three motors on the manipulator can be controlled. Two linear ironless synchronous motors drive the manipulator beam, while a slotless synchronous motor drives the arm. Incremental encoders are used to measure the position of each motor.

Each contactless technique operates in its own frequency range of the electromagnetic spectrum as is depicted in Fig. 2. The electromagnetic suspension has currents flowing in the range from DC to approximately $3 \mathrm{kHz}$, while the contactless energy transfer operates between 100 and $200 \mathrm{kHz}$. Finally, the wireless data transfer is realized by a $2.4 \mathrm{GHz}$ radio with a datarate of $1.28 \mathrm{Mbps}$. In this section, each technique is discussed in more detail.

\section{A. Magnetic suspension and propulsion}

The first challenge is the design of the magnetic suspension and propulsion that should be capable of levitating a platform with a significant mass. The planar actuator should have a unlimited long stroke movement capability in the $x-y$ plane, and short stroke in the other DOF. In addition, the contactless energy transfer should be integrated in the system, which also heavily influences the design. The high payload of the planar actuator requires larger magnets and the use of a Halbach magnet array. The coil topology is mainly determined by the design requirement to combine the two discrete functions of 


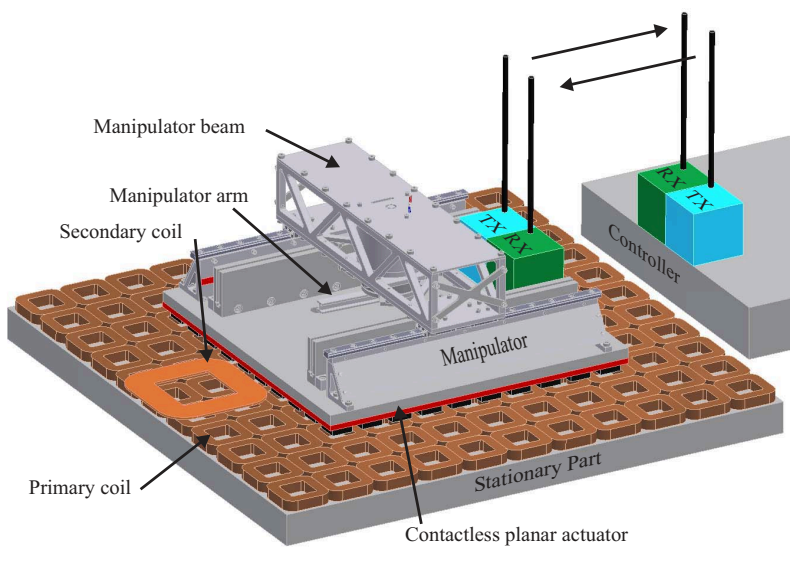

Fig. 1. Contactless planar actuator with manipulator

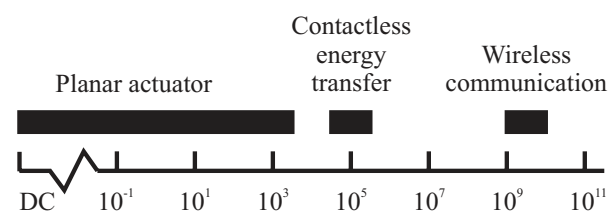

Fig. 2. Frequency range within the electromagnetic spectrum of different functions.

electromagnetic actuation and contactless energy transfer by means of the same coil set.

As discussed in [3], rectangular coils are more efficient in generating forces than square or round coils. However, rectangular coils can only produce forces in 2 directions, so a pattern of rectangular coils in two orientations 90 degrees apart is necessary to create forces in all three directions. Therefore, an array of rectangular coils does not provide sufficient coupling (higher than 20\%) between a primary coils and a single secondary coil at every position. In spite of the higher dissipation to lift a certain mass, round coils are used in this system, due to their suitability for contactless energy transfer.

The contactless energy transfer operates at frequencies in the order of $100 \mathrm{kHz}$, which requires special attention in the design of the round coils. Regular coils with solid wires have very high resistances of several $\mathrm{kOhms}$ in that frequency range due to skin and proximity effect. This can be prevented by using litz wire coils, since litz wire consists of a bundle with a number of tiny strands that are individually isolated to prevent eddy current losses at these high frequencies, thereby reducing the resistance of the coil at high frequencies to several Ohms. The disadvantage of litz wire is the lower filling factor of the coil due to the additional isolation between the strands. Regular coils with solid wire have filling factors around $70 \%$, however, for a litz wire coil, only $40 \%$ of the coil cross-section is copper. This results in higher thermal stresses.

To reduce the thermal stresses, the planar actuator platform is made using aluminum honeycomb plates that have low mass but high stiffness. In addition, the coils are mounted on ceramic discs that conduct the heat to the water cooling

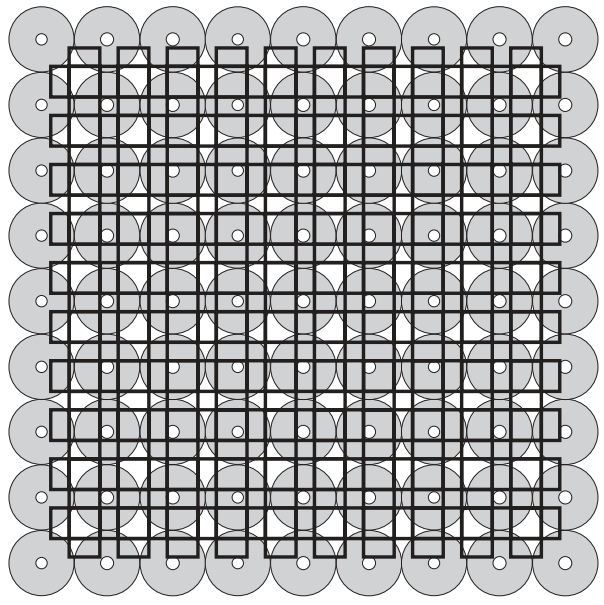

Fig. 3. Planar actuator topology

system. Finally the geometry of the coil is optimized to reduce dissipation [4].

The final design of the planar actuator consists of a Halbach magnet array with $10 \times 10$ poles, with a pole pitch of $40 \mathrm{~mm}$ and round coils, as shown in Fig. 3. The coils are shifted 240 electrical degrees and a maximum number of $9 \times 9$ coils is interacting with the magnet array at any position.

\section{B. Contactless energy transfer}

Most actuators have cables from the fixed world to the moving part to supply energy. Besides inducing disturbances such as friction and vibrations, these cables also limit the reliability of the system. Several systems already exist that can transfer energy to moving loads [9], [10], [11], [12], [13], [14], however they suffer from limited stroke or they cannot supply energy at every position. Therefore, a system should be designed that has unlimited long stroke energy transfer capability in the $x-y$ plane and that is tolerant to small changes in height (several $\mathrm{mm}$ ) and small angle variations. Finally, the system should not produce any disturbance forces and torques, otherwise it is not suitable in high-precision applications.

A secondary coil is attached to the platform next to the planar actuator, that forms an inductive coupling with one of the primary coils. A primary coil only supplies energy if it is overlapped by the secondary coil and only a single primary coil is transferring energy at a time. When it is in range of the magnet array it generates forces and torques, so these two functions are discrete and do not occur simultaneously for a given primary coil. If the secondary coil moves out of range of a primary coil, it will be switched off and the next primary coil will start transferring energy.

No iron or ferrites are used in the system, since the planar actuation is based on repulsive forces, and these materials will create attractive forces. This results in a low coupling between the primary and secondary coils. However, the geometry of the coils is optimized to minimize the position dependency of the coupling, which ensures that the energy transfer capability is fairly constant in the workspace. With an airgap of $2 \mathrm{~mm}$ the 
coupling between the coils varies between $21.9 \%$ and $24.9 \%$ in the entire workspace. To ensure a high efficiency of the energy transfer, a series resonant circuit is used that helps to boost the power supply voltage to the required $L \frac{d i}{d t}$. The power supply is a square wave voltage source that operates at the resonant frequency.

Finally, the Lorentz forces between the primary and secondary coils are in the range of several $\mathrm{mN}$ and due to the high frequency current in the order of $100 \mathrm{kHz}$, the forces oscillate above any frequency that is relevant to the mechanics.

\section{Wireless motion control}

Besides power, information is also transferred using cables and removing those cables thus requires wireless data transfer. There are several techniques available for wireless data transfer, such as bluetooth, WLAN or Ultra Wide Band (UWB). However these techniques are optimized for transferring large amounts of data and the time between sending and receiving a single packet is several milliseconds, due to the large protocol overhead. In motion control systems, the closed loop delay is usually below $1 \mathrm{~ms}$ or even less. Of course, it is possible to transfer setpoints wirelessly and run the control loop locally and then wait for several $\mathrm{ms}$ to make sure the required position is reached. Unfortunately, this approach is no longer possible if a system of multiple SISO subsystems or a MIMO system is considered. In that case more advanced controllers are needed such as position dependent feed-forward (e.g. to remove crosscoupling) or feed-back loops (e.g. gain scheduling, ). Then all systems should be synchronized in real-time.

A new wireless protocol is developed that minimizes delay using $2.4 \mathrm{GHz}$ transceivers with a datarate of $1.28 \mathrm{Mbps}$. The delay between sending and receiving of a packet with 64 bits of data is only 96 microseconds. The controller on the fixed world sends current setpoints $(3 \times 16$ bit $)$ to the manipulator and receives the positions of the motors $(3 \times 20$ bit $)$. Some of the remaining bits are used for homing and status. The custom protocol is implemented on an FPGA.

\section{EXPERIMENTAL SETUP}

An experimental setup has been realized which is shown in Fig. 4. The same features are visible as in the drawing of Fig. 1, although the primary coils have been embedded in the stator and only the black cover layer is visible. The secondary coil is attached to a power supply (with large capacitor) that converts the high frequency induced power to DC from which the motor drives (between the power supply and the manipulator) and the communication unit (on the right side) are powered.

A schematic representation of the setup is shown in Fig. 5, where each small cross is a coil that can provide forces and each circle is a coil that can transfer energy. As it is clear from this figure only six coils can provide forces and transfer energy, due to the limited range of the system in the $x-y$ plane, which is the result of the limited range of the sensors. The large thick square is the magnet array and the small thick square is

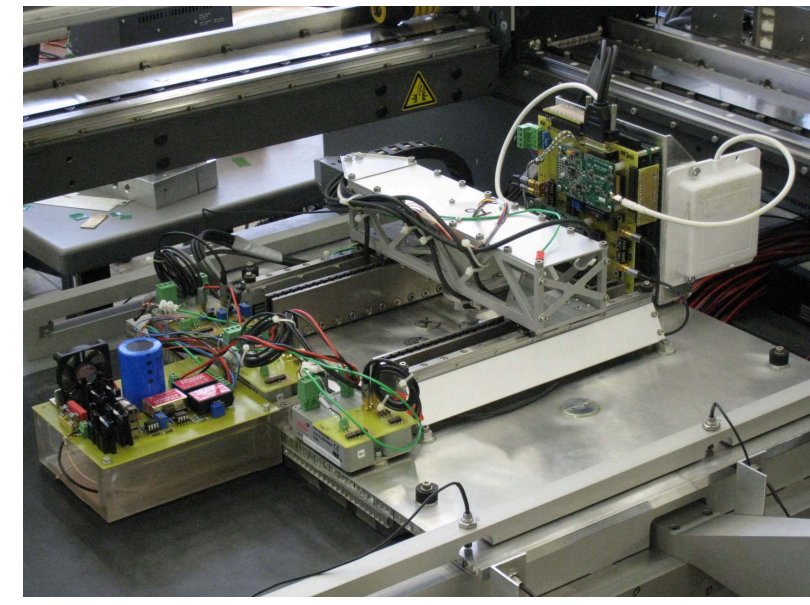

Fig. 4. Contactless planar actuator with manipulator experimental setup

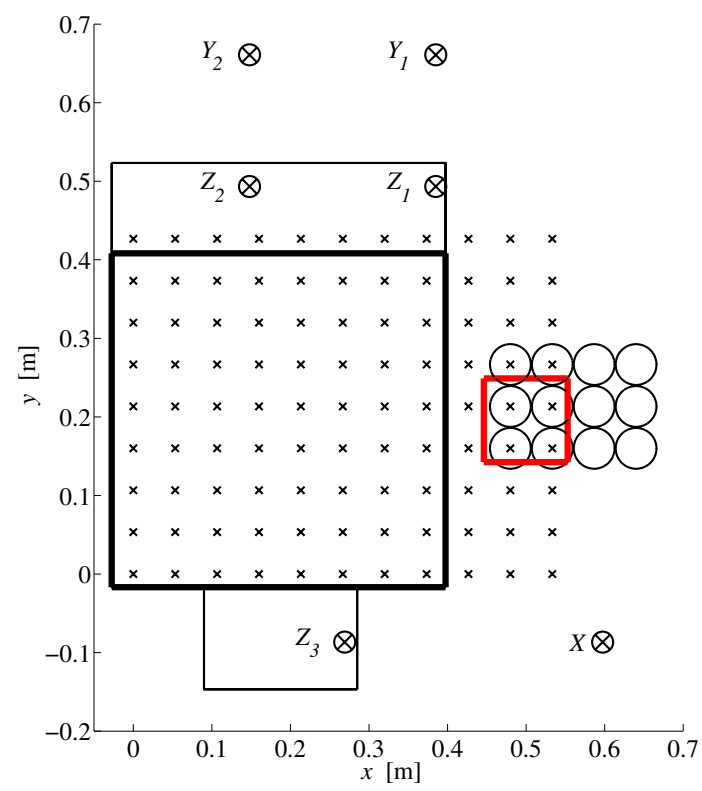

Fig. 5. Schematic representation of the experimental setup at position $x=$ $0.005 \mathrm{~m}$

the secondary coil. The thin lined rectangles attached to the magnet array are measurement references for the sensors.

\section{SENSOR SYSTEM}

The sensors are marked with an encircled cross and a label. The sensors $Y_{1}, Y_{2}$ and $X$ are long stroke laser triangulators and sensors $Z_{1}, Z_{2}$ and $Z_{3}$ are short stroke eddy current sensors. The laser triangulators have $200 \mathrm{~mm}$ range for the $X$ sensor and $100 \mathrm{~mm}$ range for the $Y_{1}$ and $Y_{2}$ sensors. The eddy current sensors have a range of $2 \mathrm{~mm}$ and are much more accurate than the laser sensors. Due to the limited range of the laser sensors, additional eddy current sensors are available besides the laser sensors.

Laser triangulators were chosen because they can measure 

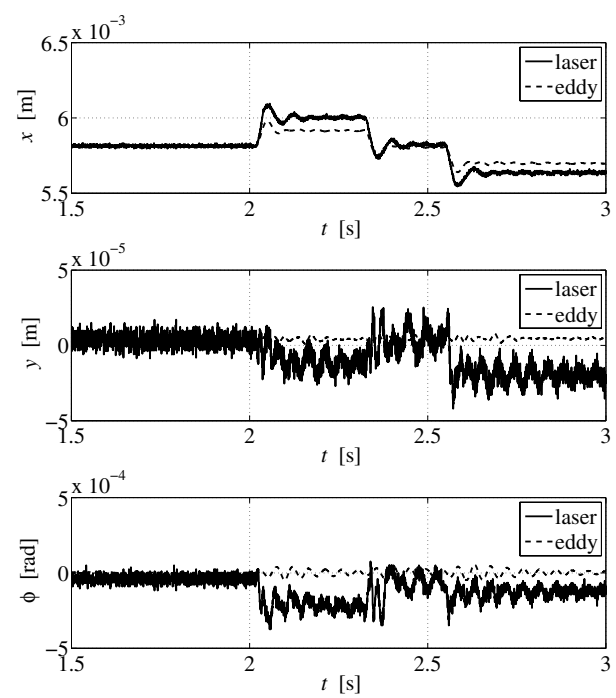

Fig. 6. Position measurements in the plane with laser triangulator sensors and eddy current sensors

distance to a surface that is not perfectly orthogonal to the laser beam. Even at angles up to 5 or 10 degrees a reliable measurement can be done, unlike laser interferometers, which of course have a much higher resolution. The commutation algorithm of this planar actuator with round coils [7] is capable of generating forces and torques with a long stroke capability in the $x$-y plane, including the rotation around the $z$-axis $(\phi)$. With laser triangulator sensors it is possible to show this, however for multi-DOF control purposes they are not suitable since they are very sensitive for changes in surface reflection. This effect is shown in Fig. 6, where a step movement in the $x$-direction causes variations in $y$ and $\phi$ positions in the measurement reconstructed from the laser triangulators. This effect is not visible if the eddy current sensors are used. Therefore, only the $x$ position is controlled using the laser sensors and the other DOF are controlled using eddy current sensors.

\section{ENERGY TRANSFER}

The energy is transferred to the manipulator using an inductive coupling at a high frequency. The square-wave high frequency driver is fed by a DC-bus power supply of which the output voltage and output current are measured. The squarewave driver has a $50 \%$ duty-cycle and runs at $158 \mathrm{kHz}$ which is equal to the resonant frequency of the primary coil including 5 meter connection cable and the resonant capacitor on the driver. This connector cable introduces significant resistance at that frequency $(7 \Omega)$ but unfortunately it is necessary to keep everything in the test setup easily accessible. In a true industrial design this cable should be omitted.

On the secondary side, the energy is stored in a $10 \mathrm{mF}$ capacitor that is kept between two voltages by a hysteresis comparator. When the upper threshold is reached the energy transfer is shut down by short-circuiting the secondary coil.
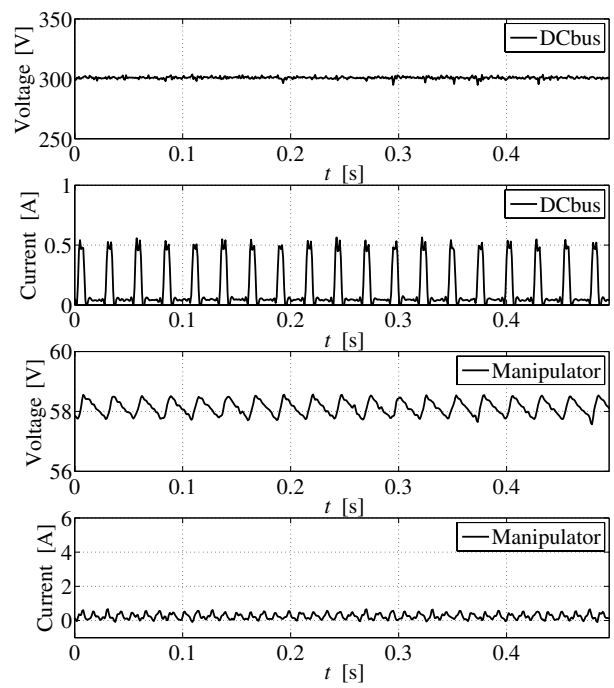

Fig. 7. Measured power input and power output of the CET system at low power
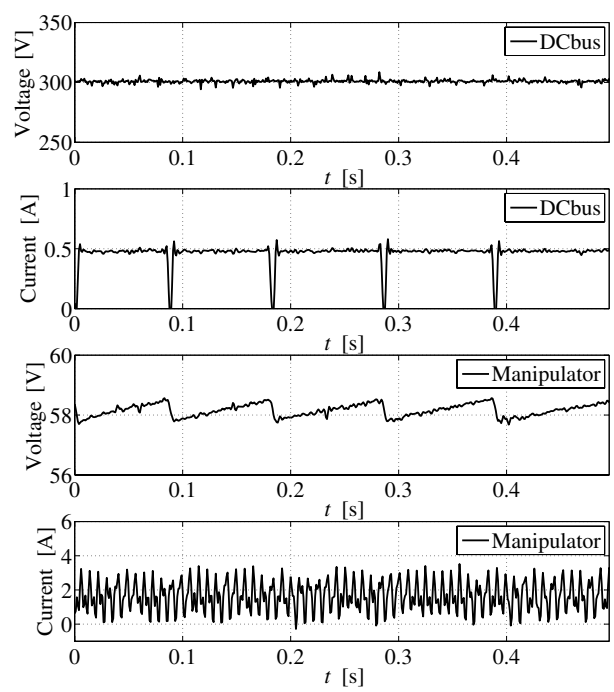

Fig. 8. Measured power input and power output of the CET system at high power

This results in a high impedance for the primary coil driver and drops the energy transfer to a few watts. When the capacitor voltage drops to the lower threshold the energy transfer is resumed. The storage capacitor is used to directly power the motor drives on the platform and to supply three DCDC converters that generate the required low voltages on the platform $(+12 \mathrm{~V},+/-15 \mathrm{~V},+5 \mathrm{~V})$ for the electronics. The power consumption of the electronics is constant $(5 \mathrm{~W})$, while the power to the motor drives is varying. This power is found by measuring the capacitor voltage and the current that flows to the motor drives from the capacitor. 


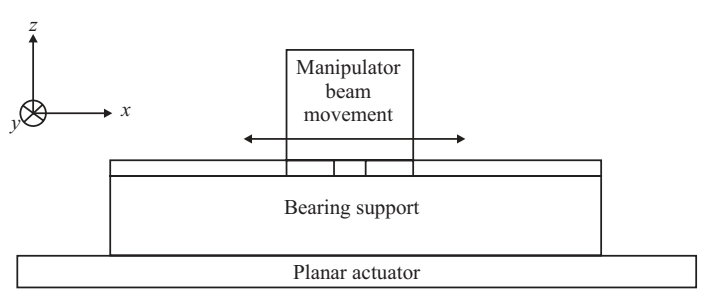

Fig. 9. Schematic 2D representation of contactless planar actuator with manipulator

The power flow is measured in two situations at low and high power output. In Fig. 7 the output to the motor drives is $15 \mathrm{~W}$ and $5 \mathrm{~W}$ to the electronics and the input power is $41 \mathrm{~W}$. The efficiency of the energy transfer is slightly less than $50 \%$. It is visible that capacitor is charged in short pulses and that the power transfer is stopped till the capacitor is discharged to the lower voltage threshold. The current to the motor drives is fluctuating, however, this fluctuations are at the switching frequency of the amplifier. Due to aliasing the variations appear to have a lower frequency.

The primary coil still draws current when no energy is transferred to the buffer which is caused by a lack of any intelligent power management but this can be included in a later stage.

When a higher output power is required, the energy transfer to the storage capacitor is mostly enabled and the discharge periods are very short, as shown in Fig. 8. The input power is $139 \mathrm{~W}$ and the total output power is $100 \mathrm{~W}$ which yields an efficiency of $72 \%$. In a previous test setup [8], where the CET system was not integrated with the magnetic suspension, efficiencies up to $90 \%$ are achieved. The reason that the efficiency is lower in this system are the losses in the long power cables, the idle power when no energy is transferred and eddy current losses in the aluminum water cooling block, which is located $15 \mathrm{~mm}$ underneath the coil.

\section{MANipulator MOVEMENT}

First, the platform is lifted at the position shown in Fig. 5 using only eddy current sensors. The commutation algorithm of the planar actuator decouples the 6-DOF, so each DOF is controlled using a PD position controller with a bandwidth of $10 \mathrm{~Hz}$. The setpoint for the controllers is a static position. The total weight of the platform is $20.5 \mathrm{~kg}$, which includes $11.5 \mathrm{~kg}$ of magnets, $2 \mathrm{~kg}$ of aluminum honeycomb material and $7 \mathrm{~kg}$ for the manipulator, power electronics, wires, etc. The torque needed to control the arm of the manipulator $\left(J=1 \times 10^{-4}\right.$ $\mathrm{kgm}^{2}$ ) is very low but the $1 \mathrm{~g}$ acceleration of the manipulator beam $(1.5 \mathrm{~kg})$ requires a significant force of the linear motors. Moreover, the beam is not located in the center of gravity of the platform as is shown in Fig. 9, so the gravity force and acceleration force create disturbance torques.

The error of the planar actuator is measured without any compensation of the reaction forces and torques, which is shown in Fig. 10, where $\theta$ is the rotation around the $x$-axis, $\psi$ the rotation around the $y$-axis and $\phi$ the rotation around the
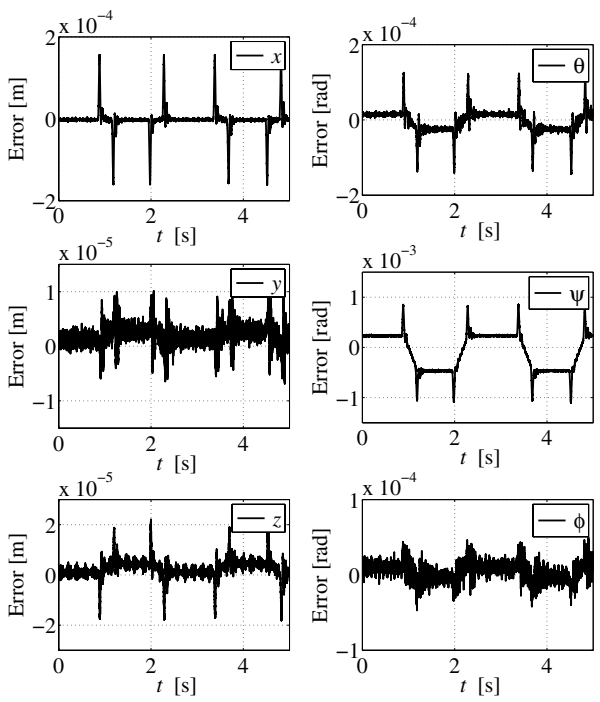

Fig. 10. Measured error of the planar actuator during movement of the manipulator without compensation
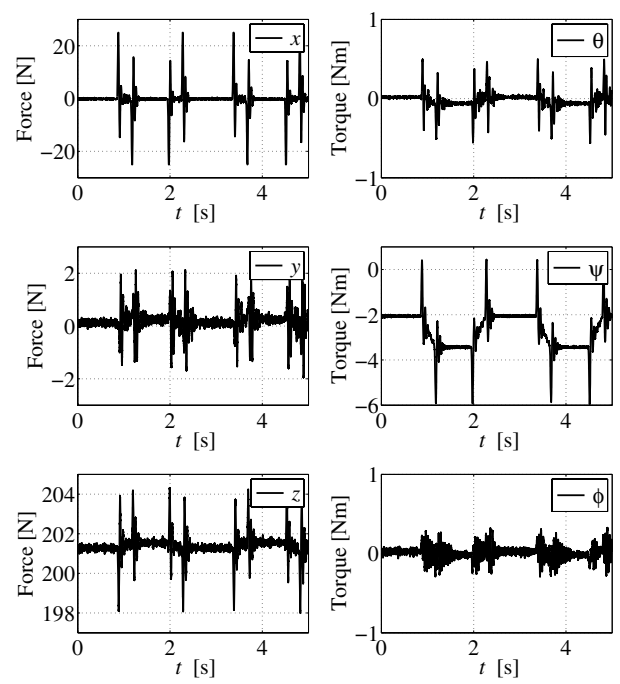

Fig. 11. Controller forces and torques of the planar actuator during movement of the manipulator without compensation

$z$-axis. The corresponding controller forces and torques are shown in Fig. 11, which clearly shows the reaction of the beam acceleration force in the $x$-direction and the changing torque around the $y$-axis due to the combined effect of acceleration force and changing position of the gravity force of the beam. Since the planar actuator is controlled using a low-bandwidth PD-controller, the errors caused by the reaction forces and torques are clearly visible.

Then the controller forces of the linear motors of the beam and the corresponding position dependent torque are used as a feed-forward in the planar actuator controllers. The effect of 

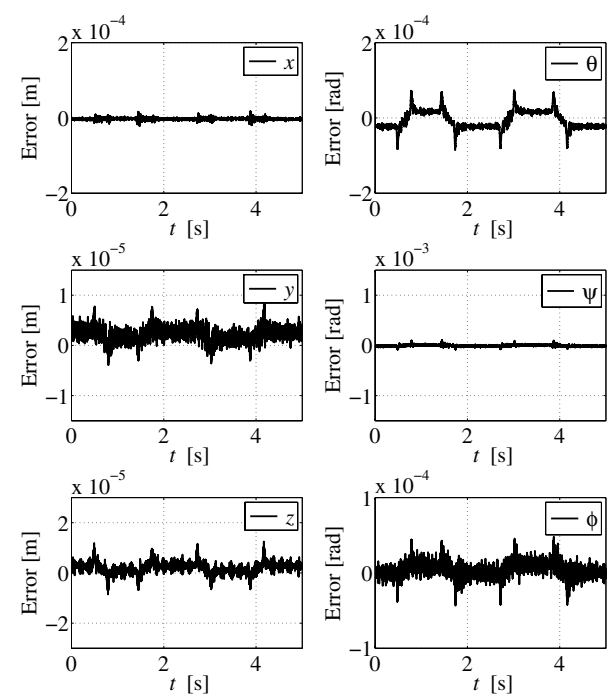

Fig. 12. Measured error of the planar actuator during movement of the manipulator with compensation
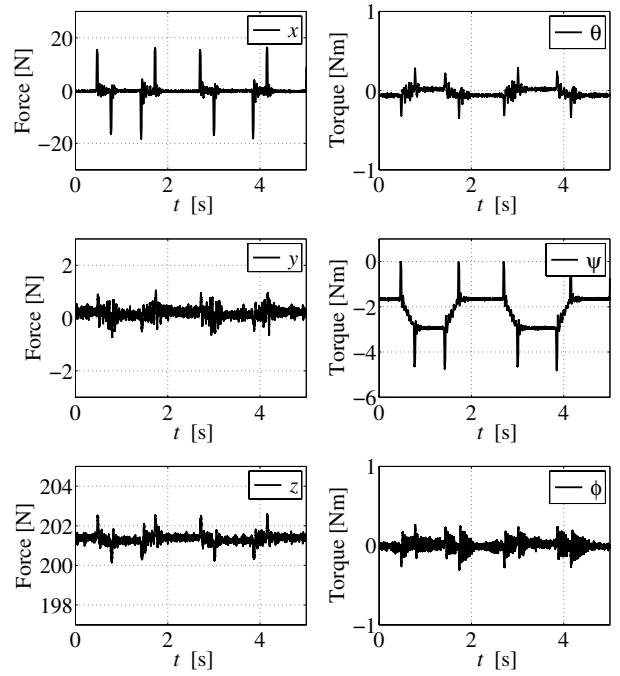

Fig. 13. Controller forces and torques of the planar actuator during movement of the manipulator with compensation

the feed-forward action on the error and the controller forces and torques is shown in Fig. 12 and Fig. 13, respectively. The errors are reduced in all directions, but especially for $x$ and $\psi$ which are the DOF associated with the disturbances. This compensation can only be done if the manipulator controller is synchronized in real-time with the planar actuator controller.

\section{LONG STROKE MOVEMENT}

The system behavior has been demonstrated at a static position without any long stroke movement of the planar actuator. The next step is to move the planar actuator in the $x$-direction in such a manner that the secondary coil will move

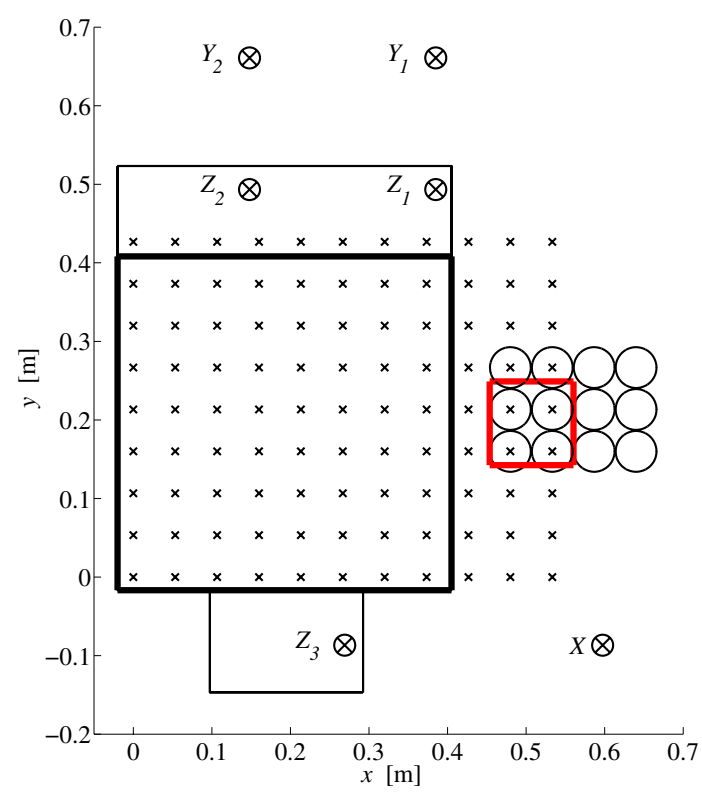

Fig. 14. Schematic representation of the experimental setup at position $x=$ $0.0125 \mathrm{~m}$

in range of a different primary coil. In Fig. 5 at position $x=0.005 \mathrm{~m}$, the coil in the middle of the left row of three (coil 86) is energized. If the planar actuator moves to the right, there is a point at position $x=0.0125 \mathrm{~m}$, where both coil 95 (which is on the right side of coil 86) and coil 86 are in range of the secondary coil as is shown in Fig. 14. The final position of the planar actuator is shown in Fig. 15, where coil 86 is out of range. The switching of the energy transfer is done with mechanical relays, so to prevent that two coils are active at the same time, a dead time of $30 \mathrm{~ms}$ is incorporated between switching one off and the other one. In addition, a dead zone of $1 \mathrm{~mm}$ in position is added to prevent continuous switching, so coil 86 is switched off and coil 96 is switched on at position $x=0.013 \mathrm{~m}$ and the opposite occurs at position $x=0.012 \mathrm{~m}$.

In Fig. 16, the measured absolute position of the planar actuator and the manipulator beam is shown, as well as the local position of the manipulator beam with respect to the platform. The manipulator beam is continuously moving between three different points with a third order position reference. The planar actuator is moved in long stroke between three $x$-positions, the other 5-DOF have a constant reference. The errors of the planar actuator during these movements are shown in Fig. 17 and the corresponding forces and torques from the controller are shown in Fig. 18.

Due to the limited resolution of the laser sensor, the overall noise levels are much higher, which is amplified by the movement of the manipulator. Although the reaction forces and torques are compensated, the amplitude of the error in $x$ direction is about the same as when the eddy current sensors are used without compensation (see Fig. 10). The errors in the other directions become larger as well, which is related to the 


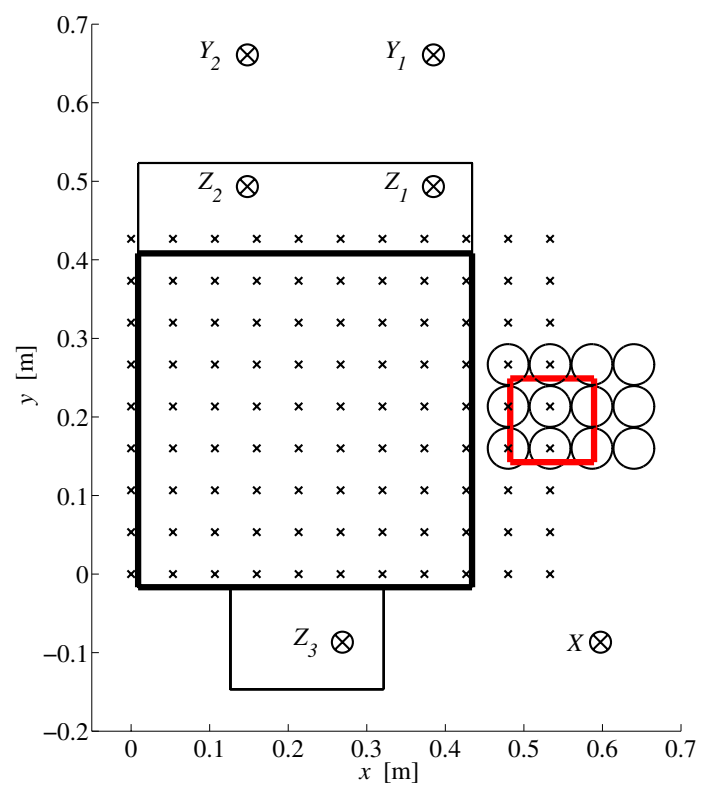

Fig. 15. Schematic representation of the experimental setup at position $x=$ $0.0415 \mathrm{~m}$
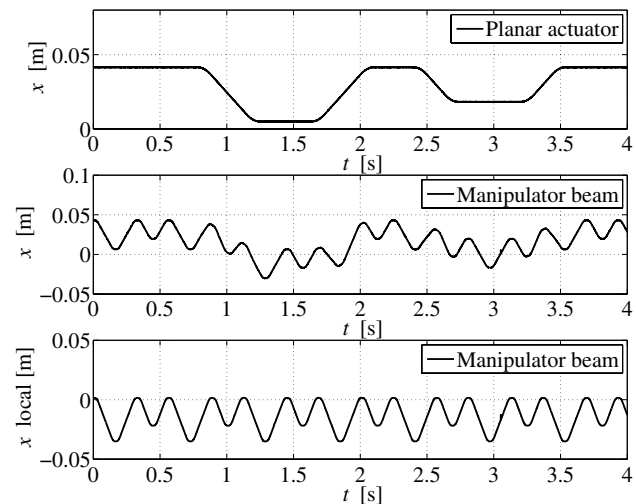

Fig. 16. Measured position of the planar actuator and manipulator beam

cross-coupling in the planar actuator. The decoupling of forces and torques is not perfect, so variations in the control force in $x$ will also influence the other forces and torques in the magnetic bearings. Finally, the error in $y$ does also depend on the $x$-position (there is no integrative action), a result of crosscoupling. This cross-coupling occurs due to limited model precision in the commutation algorithm, mechanical tolerances and measurement errors.

The flow of energy during the long stroke movement is shown in Fig. 19. Energy is drawn from the DC-bus supply during charging of the buffer capacitor on the platform. The sequences of charging and discharging are clearly visible from the capacitor voltage. The acceleration of the manipulator beam is also shown and that shape corresponds with the current drawn from the buffer capacitor. Finally, the activation
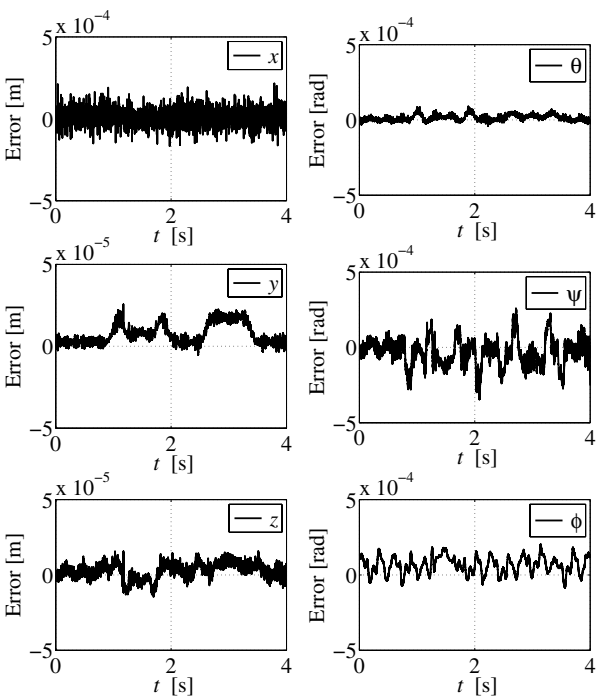

Fig. 17. Measured errors of the planar actuator during movement
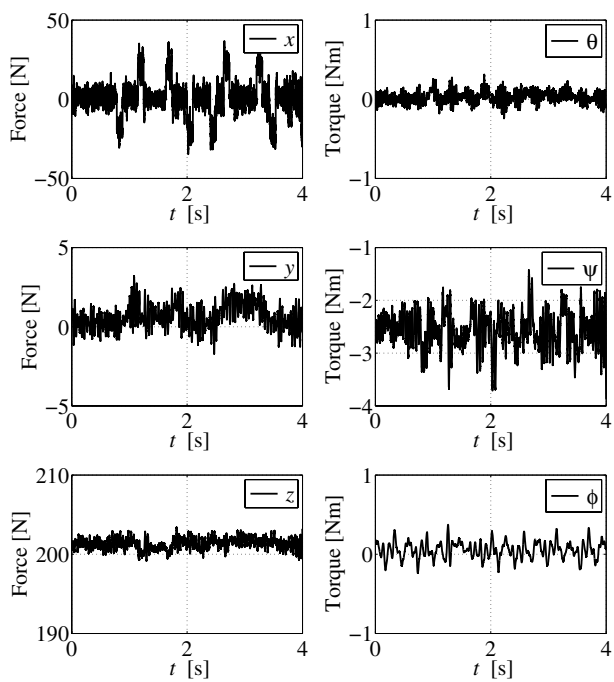

Fig. 18. Controller output of the planar actuator during movement

of the primary coils is shown, including the $30 \mathrm{~ms}$ deadtime between switch off and on. In the deadtime the buffer voltage drops to a lower value than during normal operation. The drop depends on the energy consumption of the manipulator. The first occurrence is at the same moment of maximum acceleration of the manipulator beam, and the voltage drops from $58 \mathrm{~V}$ to $55 \mathrm{~V}$. During the second primary coil transition, the manipulator beam moves at constant speed and little energy is needed, resulting in a voltage drop of $58 \mathrm{~V}$ to $57 \mathrm{~V}$.

\section{FUTURE WORK}

The contactless planar actuator with manipulator is working but there is a lot of improvement possible. The long stroke 

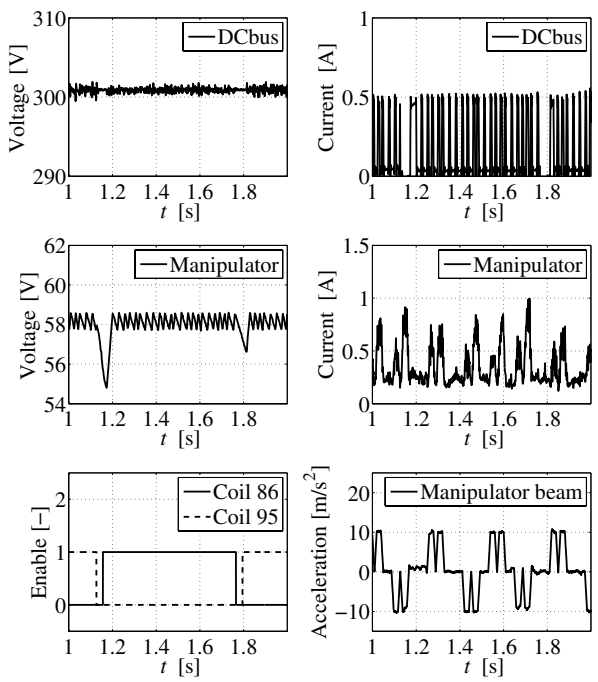

Fig. 19. Energy flow during long stroke movement

laser triangulator measurement system in the $x$ - $y$-plane, which was chosen for its ability to measure a large rotation around the $z$-axis of up to 10 degrees, turned out to give a lot of problems. Long stroke movement can only be controlled in one direction with this type of sensor. If a large rotation around the $z$-axis is not important, the measurement system can be replaced with a laser interferometer based system. Otherwise, a new measurement system needs to be developed.

Another problem is the implementation of the contactless energy transfer system. Due to time and budget restraints, a mechanical relay is used to switch the coil from the current amplifier to the high frequency energy transfer driver. This mechanical relay is very slow, resulting in long switching deadtimes, which interrupt the energy transfer. A more elegant solution would be to combine the current amplifier and the energy transfer driver into one system. The power stage of a PWM based current amplifier should be able to switch at frequencies in the same range as the energy transfer $(<$ $200 \mathrm{kHz}$ ). If the unit acts as an energy transfer driver, it will change to a $50 \%$ duty-cycle square wave voltage source. This requires a reprogramming of the FPGA-logic that is available in most modern current amplifiers.

Finally, the energy transfer drivers could be placed close to the coils to limit losses and EMC.

\section{CONCLUSION}

In this paper, a novel motion system is presented that consists of a 6-DOF magnetically levitated planar actuator platform with long stroke movement capability in the $x-y$ plane. On top of the floating platform is a manipulator that can operate without any cables from the fixed world. The energy to the platform is supplied by means of an inductive coupling between the stationary and the moving part with long stroke contactless energy transfer capability. The control of the manipulator is done by means of a wireless real-time data link. All controllers are on the fixed world, which allows for real-time synchronization of the total system.

The behavior of the system is demonstrated with measurements. Besides its application together with a 6-DOF planar actuator, the contactless energy transfer system and wireless motion control can be applied in any motion system, where cables are unwanted.

\section{REFERENCES}

[1] A.J. Hazelton, M.B. Binnard and J.M. Gery: Electric Motors and Positioning Devices Having Moving Magner Arrays and Six Degrees of Freedom, US Patent 6,208,045, March 2001.

[2] J.C. Compter: Electro-Dynamic Planar Motor, Precision Engineering, Vol 28, Issue 2, pp. 171-180, April 2004.

[3] J.W. Jansen, C.M.M. van Lierop, E.A. Lomonova and A.J.A. Vandenput: Modeling of Magnetically Levitated Planar Actuators with Moving Magnets. IEEE Trans. on Magnetics, Vol. 43, No. 1, pp. 12-25, May 2007.

[4] J.W. Jansen, C.M.M. van Lierop, E.A. Lomonova, A.J.A. Vandenput, Magnetically levitated planar actuator with moving magnets, Proc. IEEE Int. Electric Machines and Drives Conf. (IEMDC'07), Antalya, Turkey, May 2007, pp.272-278.

[5] C.M.M. van Lierop, J.W. Jansen, A.A.H. Damen and P.P.J. van den Bosch: Control of Multi-Degree-of-Freedom Planar Actuators, IEEE International Conference on Control Applications, Munich, Germany, pp. 2516-2521, October 2006.

[6] J. de Boeij, E. Lomonova, A.J.A. Vandenput: Modeling Ironless Permanent-Magnet Planar Actuator Structures, IEEE Trans. on Magnetics, Vol. 42, No. 8, pp 2009-2016, August 2006.

[7] J. de Boeij, E. Lomonova, and A.J.A. Vandenput: Look-Up Table Based Real-Time Commutation of 6-DOF Planar Actuators, IEEE International Conference on Control Applications, Singapore, October 2007, CDROM.

[8] J. de Boeij, E. Lomonova, J.L. Duarte, A.J.A. Vandenput, Contactless Energy Transfer to a Moving Actuator, IAS 2006 Annual Meeting, Tampa, FL, USA, October 2006, CDROM.

[9] G.L.M. Jansen: 2-Dimensional Displacement Device, International Patent Application, WO 2005/013464 A1.

[10] G.A. Covic, G. Elliott, O.H. Stielau, R.M. Green, J.T. Boys, The Design of a Contact-less Energy Transfer System for a People Mover System, Proceedings IEEE International Conference on Power System Technology, PowerCon 2000, Vol 1, December 2000, pp. 79-84.

[11] H. Ayano, K. Yamamoto, N. Hino, I. Yamato, Highly-Efficient Contactless Electrical Energy Transmission System, 28th Annual Conference of the IEEE Industrial Electronics Society, IECON 2002, Vol 2, November 2002, pp. 1364-1369.

[12] S. Adachi, F. Sato, S. Kikuchi: Considerations of Contactless Power Station with Selective Exitation to Moving Robot, IEEE Transactions on Magnetics, Vol. 35, No. 5, September 1999, pp. 3583-3585.

[13] F. Sato, J. Murakami, H. Matsuki, S. Kikuchi, K. Harakawa, T. Satoh: Stable Energy Transmission to Moving Loads Utilizing New CLPS, IEEE Transactions on Magnetics, Vol. 32, No. 5, September 1996, pp. 5034-5036.

[14] F. Sato, H. Matsuki, S. Kikuchi, T. Seto, T. Satoh, H. Osada, K. Seki: A New Meander Type Contactless Power Transmission System - Active Excitation with a Characteristics of Coil Shape, IEEE Transaction on Magnetics, Vol. 34, No. 4, July 1998, pp. 2069-2071. 